\title{
NeuroCar Virtual Driving Environment: Simultaneous Evaluation of Driving Skills and Spatial Perceptual-attentional Capacity
}

\author{
H. Hämäläinen ${ }^{1}$, F. Rashid Izullah ${ }^{1}$, A. Aho ${ }^{1}$, M. Koivisto ${ }^{1}$, T. Laine ${ }^{1}$, \\ P. Qvist ${ }^{2}$, A. Peltola ${ }^{2}$, P. Pitkäkangas ${ }^{2}$, M. Luimula ${ }^{2}$ \\ ${ }^{1}$ University of Turku, Centre for Cognitive Neuroscience, \\ Turku Brain and Mind Center \\ Assistentinkatu 7, 20014 University of Turku, Finland \\ Phone: +358 400711364 \\ e-mail: hhamalai@utu.fi \\ ${ }^{2}$ Turku University of Applied Sciences, Turku Game Lab \\ Joukahaisenkatu 3, 20520 Turku, Finland
}

Abstract: We describe here a simple, inexpensive and effective system for simultaneous evaluation of a subject's driving ability and spatial auditory and visual perception and attention. It consists of a commercial steering wheel and virtual glasses and a program for driving on a two-lane road with curvatures at about $100 \mathrm{~km} / \mathrm{h}$ speed, and simultaneously reacting by pressing two buttons attached to the steering wheel to randomly delivered uni- and bilateral auditory signals via earphones and light dots appearing in the peripheral visual field. Three different difficulty levels of the task were applied in randomized counterbalanced order, each session of $2 \mathrm{~min}$ duration. The results of 25 young (17-45 years) and 20 elderly (47-96 years) healthy participants demonstrate the tendency for simultaneous right side spatial perceptual/attentional bias and the left side driving bias especially in the elderly participants.

Keywords: driving ability, spatial perception, attention, bias, age

\section{Introduction}

We have shown in our previous studies that humans are hardwired to a rightward bias in spatial perception and attention - this concerns at least most right-handers [1-3]. This bias is strongest in children and elderly, but occurs also in young when under cognitive stress. We have presented a hypothesis and proposed a model, based on latest findings in cognitive neuroscience, according to which this age- and cognitive load-dependent spatial bias is due to the lateralization of attention mechanisms in the human brain, and slow development in the young and later decline in the old by the executive functions 
which are responsible for our skills for cognitive control, e.g. (in)voluntary attention, working memory, and purposeful, goal-directed behavior. The changes of the spatial bias as a function of the lifespan - distinct in childhood and old age - well reflects the interplay between the attentional-cognitive control mechanisms [4].

Since we only studied this bias in laboratory the question remains, whether this bias can also be demonstrated in more ecologically valid conditions, i.e. traffic. Therefore we constructed a simple system with steering wheel with inserted right and left buttons for responses. Virtual 3-D environment for the driving task was created with virtual glasses (Oculus Rift) and visual stimuli were applied to the periphery of the visual field and auditory stimuli were delivered via earphones. With this system it is possible to mimic and even replicate the previous laboratory experiments [2, 3] simultaneously while the subject is driving the imaginary car in along the road and in different driving conditions. This simple system allows us to determine the subject's capacity to 1) drive (keep the vehicle on the road/lane), respond to the auditory and visual spatial stimuli while driving, and the mutual effects of these two tasks on each other at different difficulty levels.

With this system we aim at an international database-based evaluation platform, where one can very quickly screen whether the subject in case drives and perceives the spatial stimuli properly according to the age, handedness, gender, etc. specifications. If not, the system warns the experimenter, and the subject needs more specific examination.

Based on thorough piloting we ended up in running three different difficulty levels to participants from all age levels. This is due to the fact that the variability in spatial perception, speed, and (quite unexpectedly) the speed and familiarity in button press technique is today so large, that if having only one difficulty level we would have ended with numerous younger participants with a ceiling effect and numerous older participants with interruption of the task because of impossibility of accomplishing the task as the other extreme.

In this report we will describe the properties and application of the system and the first results on effects of age on driving performance and simultaneously measured spatial attention perception and attention. Because we also wanted to check the occurrence of the right bias in our elderly participants, only right-handed participants were included in the present population.

\section{Methods}

\subsection{Apparatus and stimuli}

\section{NeuroCar system}

NeuroCar system was first introduced in (Luimula et al., 2015). Our multidisciplinary team has developed the first prototype of the virtual evaluation tool to be used for driving acuity and spatial perceptual capacity test in a virtual environment. This prototype consists of Oculus Rift Development Kit 2 data glasses, off-the-shelfheadsets, Fanatec Porsche 911 GT3 RS V2 Wheel US attached with Arduino response buttons, and software developed with Unity 5 game development platform. Oculus Rift 
data glasses has been used for 3-D presentation of the road and spatial peripheral visual stimuli, and headsets for presenting spatial auditory stimuli (the data glasses can be replaced for certain purposes by video screen).

The developed prototype has a user interface designed for the test subject but it offers for the researcher also administrative features. The difficulty level of the driving and spatial perception can be controlled and adjusted to the demands of the performance level of the driver (can be compared against normative data in a large international database later, backend system explained later in this chapter). Parameters to be varied are at the moment a speed of the vehicle, a rate of the spatial stimulus application, a visibility of the road, difficulty of the road (curvature, and width), and intensity of the stimuli (simultaneous evaluation of functioning of the visual and auditory systems). At the moment, administrative panel contains also features that will help to establish right parameters for the final product to simulate realistic driving experience.

Driving acuity can be determined during the driving task itself. Our virtual evaluation tool measures the rate and amount of driving errors (i.e. driving out of lane, direction of errors, and time spent outside the lane), where it is possible to assess the level of driving performance and the possible bias to the left or right. Also the relation of the driving errors to the spatial visual and auditory stimuli will be determined (the induction of the errors by the spatial stimuli, e.g. whether the response to right stimuli induces driving error to the right or left).

Spatial perceptual capacity can be determined during the driving with controlled series of visual and auditory peripheral spatial stimuli in separate trials or mixed together. With the very same stimulus series it has been shown distinct deterioration and biasing of the perceptual abilities in the healthy elderly population in laboratory conditions without any background driving task. The participant's task is to respond as quickly as possible to stimuli by pressing the left or right button (attached to the steering wheel) or both, to stimuli delivered from the left or right direction or from both simultaneously, correspondingly. The accuracy of responses and reaction times will be determined. This the most objective way of determining the degree of perceptual biases in the driver's perceptual capacity.

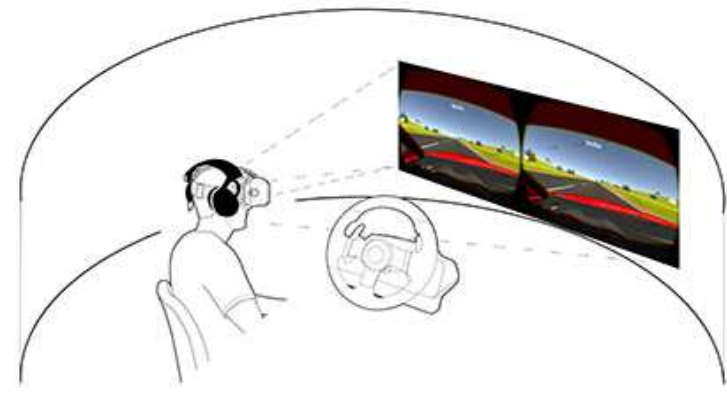

Figure 1. System description of the NeuroCar system in this study.

NeuroCar version reported in this paper is designed only for driving inspections so far. The training environment is at the moment under development. In this study, the driver is steering a virtual car which can be steered with a haptic steering wheel. The 
test subject (the driver) has headsets which will simulate 3D auditory environment. In addition, the test subject has Oculus Rift data glasses which will show for the driver virtual environment including road, simple terrain, and visual signals. While driving the test subject has to react on visual and auditory disturbances by pressing buttons attached to the steering wheel.

In the current prototype (Figure 1), driving environment is visualized in Oculus Rift data glasses which enables an immersive driving feeling. The camera view is showing the driving view from the car inside (Figure 2). The driver is able to see $3 \mathrm{D}$ virtual environment. Data glasses are reacting on the driver's head movements to increase the immersion. Visual disturbances are visualized peripherally 50 milliseconds and the driver has to react on those by pressing buttons attached to the wheel (defined in the system as mouse buttons). Similarly the driver has to react on auditory disturbances by pressing the buttons while hearing 3D sound stimuli from the headsets.

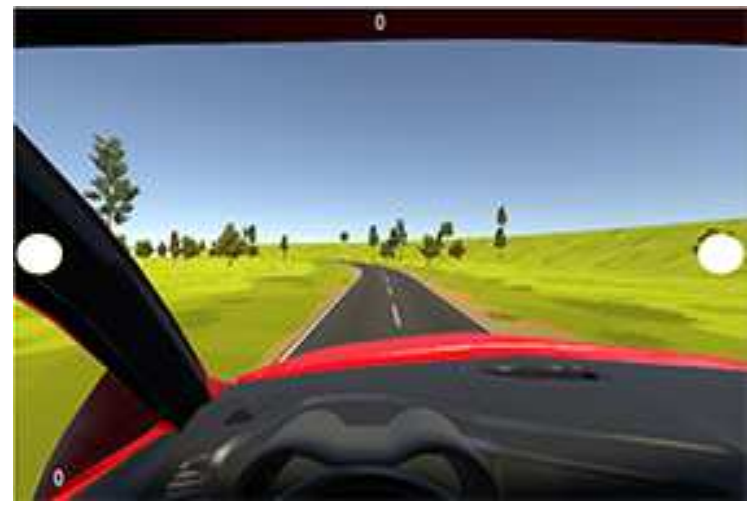

Figure 2. A screen capture of drivers's perspective in Oculus view.

The buttons has been implemented attaching external buttons to the steering wheel controller. By pressing these buttons left or right mouse buttons clicks will be activating. These buttons has been situated ergonomically close to the existing game buttons so they will be easy to press while driving. The demonstration application is able to record signals from the buttons in the log file which is consisting successful button clicks together with the time stamp in milliseconds. The log file data is currently in textual format so later in the project all the information will be stored in the database in the back-end system.

\section{Backend system}

The driving inspection and the future training environment systems have been designed to benefit from an automatized background data gathering system for purposes of research supporting analytics, personal progress tracking, and future opportunities in official or medical use. The data gathering system was created around the concept where the end user could track individual inspection cases with a high level of detail, allowing the test organizer (e.g. doctor) and test subject to play back replays of driving evaluation tests or training rounds. The system has also been designed to record any significant events during the simulation, such as events prompted to the end user to 
react upon, events of hazards or possible hazards (e.g. near-collision situation), and trackable driving situation events such as changing lanes or reacting to traffic signs.

The background system is based on a database capable of storing large amounts of information, thus a modern cloud based solution was selected as a primary platform already in the preliminary phase of the system architecture design. The database was designed to collect personal background information, basing the system on the existing pen-and-paper method of background data collecting, and extending the requirements based on discussions with the researchers from the research team. This background data involves non-identifiable personal information (e.g. gender and age), medical information (e.g. medicine use, eyesight, sleeping habits) and video game playing experience and habits. The second portion of the database is structured to store detailed information of all the data linked to the personal information data, so the database is designed to host data collections of medical substances, drugs, disorders and other medical conditions. This structure of data will allow gathering the personal background data in systematic and normalized manner. The third portion of the database structure will host large datasets to store all the driving test and training recordings. With each recording, personal background condition fields can be attached, for example allowing the system to store data of how many hours the person has slept before the recording, or if there has been medicine or other substance used within the past 24 hours.

The personal background data entry is done digitally, for example by the test arranging personnel or the test subject, employing easy-to-use entry methods, for example with digital forms on a tablet computer. User interface and usability design will be employed to ensure a very low error level in the data entry process. The current twostep system of pen-and-paper entry, which is then stored on a computer system presents an extra step which can act as an entry point for human error in systematic data gathering, and the newly designed digital system is based on the concept of minimizing the effect of human error in any data entry steps.

The driving simulation recording system is fully automated, and after the background data entry steps, the person attending the simulation will need no extra effort or attention for the data gathering to be executed. This recording system works in similar manner as in driving video games, where the whole session is recorded in the working memory of the computer, allowing instant playback of the simulation session, and furthermore storing the session data into the analytics database. The gathered session data structure is designed to host virtually all possible conditions and events happening during the simulation, thus allowing analytics in the future to research any circumstances that might have occurred within the simulation. Consequentially, this recording and playback engine allows researchers in the future to tap into vast datasets of driving simulation data gathered, and allows research on various topics, which might not even have been identified yet today. This form of vast datasets, or big data, creates a system that in the future will allow data analytics to find new patterns of data, for the purpose of medical research, official and regulatory use (e.g. police or traffic officials), and the data itself will become a valuable resource, which creates additional benefit and added value upon the training and test benefits of the simulation system. 


\section{Backend data security considerations}

When storing sensitive personal data, such as medical information or substance use habits, even a local database would need a firm scrutiny of data security procedures involved within the whole system. When employing a cloud based server system for hosting the background and analytics database, the data security questions arise to a whole another level. Not only the database itself needs to follow strict data security guidelines, but all the data traffic between the simulation and background data gathering clients and the cloud servers need to be secure and encrypted.

In the preliminary data architecture design phase, discussions with the current stage of the cloud system data security were conducted, and the conclusion was that as long as personally identifiable data is not stored into the database, the data security level of current cloud systems is adequate for the background and analytics data gathering of the designed system. Furthermore, the reliable and big cloud system operators host their server computers in data centers, which have strict data security protocols, up to the level of having guards and restricted entry to the facilities itself. The digital data security level of the modern cloud systems most often is of higher level than an individual research organization could provide, with $24 / 7$ data security support and administration provided by the cloud operator.

For future needs, the personally identifiable data, such as name or social security number can be hosted in another database, of higher level of security, and the identifier of personal information will be tokenized within the analytics and background data database. This tokenization ensures, that personal information can be revealed only oneway, and with officials (e.g. medical doctor or police) who have access to the personally identifiable data database, with higher level of data security policies. The personal information data cannot be revealed the other way around from the side of the background and simulation recording database, thus allowing a secure architecture to store sensitive data. This method of tokenization of personal identifiers will be designed during the research project, however for the prototype or proof-of-concept level system to be implemented during the project, the tokenization and personally identifiable data will not be stored into the database. This allows manageable data security level to be implemented during the research project, but the design can be in the future expanded to a more strict data security level solution via the tokenization methodology.

\subsection{Participants}

The participants were right-handed young and elderly adults. The young adult group consisted of 25 individuals (17-45 years of age; 14 female), and elderly group consisted of 20 (47-96 years of age; 11 female). The right-handedness was chosen here as an inclusion criterion, because our previous findings on spatial perceptual/attentional bias is based in right-handed population implicating a certain type of lateralization of brain functions [4]. Seniors mostly were recruited from foster (old-age, nursing) homes and elderly recreational clubs/facilities, whereas the young adults were mostly university students.

The participant had to be physically healthy with no neurological or psychiatric problems. Corrected vision and hearing were approved. 


\section{Procedure}

\subsection{Questionaires}

The tests of the study partly conducted at the Centre for Cognitive Neuroscience, University of Turku. For the convenience of the elderly, the tests when necessary were conducted in the natural environment of the participants like recreational locations and old-age homes. Before the tests three forms were to be filled by the participant. First one was targeted usage of drugs and alcohol, hours of sleep, and level of alertness/tiredness at the time of testing. Also issues such as profession, driving experience and accidents, the experience with computer games etc. were asked. The second inquiry evaluated handedness. Third one focused more thoroughly on participants' game habits, and was filled only if the participant actively played computer games.

\subsection{Driving Tasks}

The driving task was adjusted (via a long series of piloting with drivers of different ages) to mimic driving on a normal countryside two-lane road with hills and curvatures and with a speed of about $100 \mathrm{~km} / \mathrm{h}$, and was considered rather easy for a person with usual driving experience. The most problematic part in driving task was the steering wheel - most manufacturers produced steering wheels that mimicked those for racing cars (F1), and thus were absolutely too different from the steering wheels of ordinary cars what comes to the feel of the steering wheel and the driving itself. After a long series of trials we ended with the steering wheel by Fanatec Porsche 911 GT3 RS V2 Wheel US (TM). The steering experience must be as close to the ordinary, because otherwise the unfamiliarity of the steering itself takes over too much of the driver's attentional capacity. This also is a challenge to manufacturers of the steering wheels for games.

The errors in driving, i.e. crossing the lane border, time spent on or outside the lane border, and the correction of errors were all stored to allow many types of error analyses. Here we only report number and side of crossing the lane border during the session.

\subsection{Stimuli and measures of performance}

The visual stimuli were white dots appearing in the periphery of the visual view in the lower left and right quadrants of the visual field (see Fig. 2). The location of the dots was constant (but can be varied for instance in applications for patients with blind spots in their visual field).

The auditory stimuli, applied via headphones (SHURE/SRH440) were short sine wave bursts of $550 \mathrm{~Hz}$ frequency and $50 \mathrm{~ms}$ duration, and enveloped to avoid the overshoot (clicks) in the beginning and the end of the burst. The intensity was adjusted to be $66 \mathrm{~dB}$, and adjusted for each participant individually whenever necessary.

The auditory and visual stimuli were applied unilaterally to the left, right, and bilaterally in randomized order, and the participant's task was to react to the stimuli as quickly as possible by pressing corresponding button attached to the left and right sides 
of the steering wheel. Three different difficulty levels, high (ISI 500-1000 ms varying in steps of $13 \mathrm{~ms}$ ), medium (ISI 700-1200 ms), and low (ISI 1000-2000 ms) were chosen after piloting of the system with participants of different ages. Some of the young participants showed $100 \%$ correct responses, i.e. ceiling effect, at the lowest difficulty level, whereas some of the old participants had zero correct responses at the difficult level. The correctness of the response (occurring within 150-1000 ms after the stmulus inset) and reaction times (RTs), i.e. speed of the correct responses, were determined as performance indicators.

\subsection{Training and test sessions}

The test sessions were always preceded by pilot runs, where the participants first got acquainted with the driving task and the steering wheel ( $2 \mathrm{~min})$. Then a training session with just the visual and auditory stimuli and responding to them by button presses but without driving task ( 2 min) was accomplished. Finally, the driving task was combined with the stimuli and button presses ( $2 \mathrm{~min})$.

After the training the test sessions were run each of the difficulty levels twice in counterbalanced order (ABC-CBA). The order of sessions was randomized for each participant. The duration of each test session was adjusted to be $2 \mathrm{~min}$, which resulted in average numbers of both auditory and visual stimuli (uni- and bilateral together) being 24-25 in the fast (ISI 500-1000 ms), 18-20 in the medium (ISI 700-1200 ms), and 12-13 in the slow (ISI 1000-2000 ms) stimulus spacing, on the average. The order of auditory and visual stimuli and stimulus conditions were randomized. The participant's key task was to keep the vehicle on the lane, but also to detect as many stimuli as possible.

\section{Results}

\subsection{Driving errors: Crossing over the lane borders}

Fig. 3 shows the driving errors, i.e. crossing over the lane borders to right or left by the two age groups at the three task difficulty levels. Three-way mixed ANOVA [Factors: driving error side (left, right), difficulty level (fast, medium, slow), age (young, elderly)] applied to average percentage of errors to the left and right side crossings, resulted in significant main effects for driving error side, $F(1,41)=6.73, p=$ 0.01. Participants crossed the left lane border more than the right (Fig. 3).

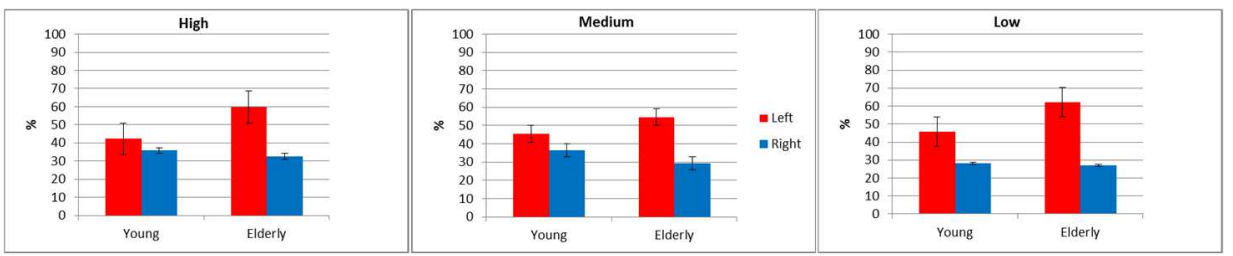

Figure 3. Driving errors (lane border crossing) by the two age groups at the three difficulty levels (spacing of the spatial stimuli), high(ISI=500-1000 ms), medium(ISI=700-1200 ms), and low(ISI=1000-2000 ms) 


\subsection{Auditory stimuli}

\section{Correct responses}

Fig. 4 shows correct responses by the two age groups to three auditory stimulus conditions at three difficulty levels. Three-way mixed ANOVA [Factors: difficulty level (high, medium, low)* stimulus condition (left, bilateral, right)*age (Young, elderly)] revealed significant main effects for stimulus condition, $F(1,44)=30.89$, $p<0.01$, difficulty level $\mathrm{F}(1,61)=174.30, \mathrm{p}<0.001$, and age, $\mathrm{F}(1,41)=10.42, \mathrm{p}<0.01$. The young in general outperformed the elderly. There was a significant interaction between difficulty level and stimulus condition, $\mathrm{F}(3,108)=4.10, \mathrm{p}=0.01$. The effects were further investigated with pairwise comparisons using Fisher's LSD tests. Participants gave a smaller number of correct responses at the high $(\mathrm{M}=40)$ than at the medium $(\mathrm{M}$ $=61)$ and at the low $(\mathrm{M}=75)$ difficulty levels [high-medium $(\mathrm{p}<0.01)$, high-low $(\mathrm{p}<$ $0.01)$, medium-low $(\mathrm{p}=0.01)]$. At the high difficulty level, the participants gave more correct responses to the right $(\mathrm{M}=47)$ than to the left $(\mathrm{M}=44)$ stimuli $(\mathrm{P}<0.01)$. At the medium difficulty level, more correct responses were given to the right $(\mathrm{M}=70)$ than to the bilateral $(\mathrm{M}=45)$ stimuli $(\mathrm{p}<0.01)$. At the low difficulty level, more correct responses were given to the left $(\mathrm{M}=83)$ than to the bilateral $(\mathrm{M}=58)$ stimuli $(\mathrm{p}<$ 0.01) (Fig. 4, bottom).

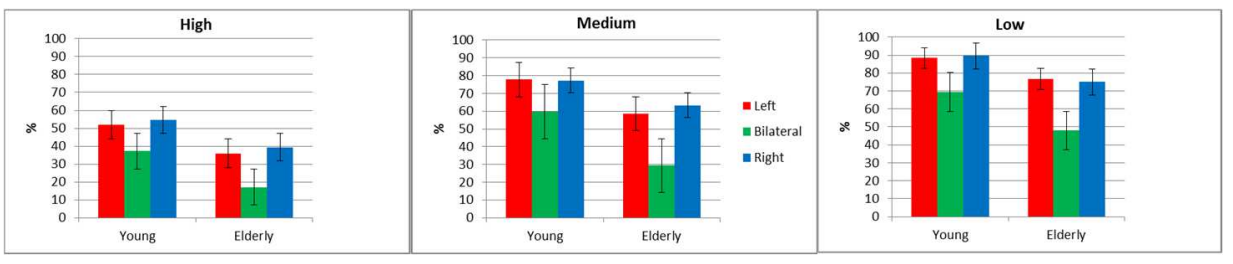

Figure 4. Correct responses in the three auditory stimulus conditions, left, bilateral, and right, by the two age groups at the three difficulty levels, High(ISI=500-1000 ms), medium(ISI=700-1200 ms), and low(ISI=1000-2000 ms).

\section{$R T s$}

Figure 5 shows the RTs of the two age groups at the three difficulty levels in the three auditory stimulus conditions. Three-way mixed ANOVA [Factors: difficulty level (high, medium, low)*stimulus condition (left, bilateral, right)*age (young, elderly)] revealed significant main effects for difficulty level, $\mathrm{F}(2,68)=58.16, \mathrm{p}<0.01$, stimulus condition, $\mathrm{F}(2,50)=43.21, \mathrm{p}<0.01$, and age, $\mathrm{F}(1,34)=6.90, \mathrm{p}=0.01$. The elderly in general were slower than the young. An interaction between difficulty level and stimulus condition, $\mathrm{F}(3,86)=13.36, \mathrm{p}<0.01$, was detected. Further analysis using Fisher's LSD pairwise comparisons was conducted. Significantly longer RTs were obtained at the high $(\mathrm{M}=570)$ difficulty level followed by low $(\mathrm{M}=663)$ and medium $(\mathrm{M}=635)$ difficulty levels [high-medium $(\mathrm{p}<0.01)$, high-low $(\mathrm{p}<0.01)$, medium low $(\mathrm{p}<0.01)]$. At the medium difficulty level, the participants reacted faster to the right $(\mathrm{M}$ $=603)$ than to the left $(\mathrm{M}=626)(\mathrm{P}<0.01)$ stimuli (Fig. 5, middle). At the low difficulty level, the participants reacted faster to the right $(\mathrm{M}=619)$ than to the left $(\mathrm{M}$ $=633)(\mathrm{p}=0.02)$ or than to the bilateral $(\mathrm{M}=731)(\mathrm{p}<0.01)$ stimuli $($ Fig. 5 , bottom $)$. 


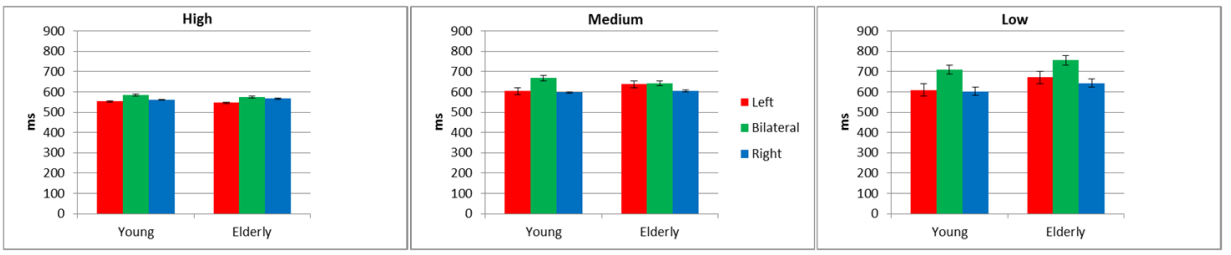

Figure 5. Reaction times in the three auditory stimulus conditions, left, bilateral, and right, by each group at the three difficulty levels, high(ISI=500-1000 ms), medium(ISI=700-1200 ms), and low(ISI=1000-2000 ms).

\section{Erroneous unilateral responses to bilateral stimuli}

Fig. 6 shows erroneous unilateral responses in the bilateral stimulus condition by the two age groups at the three difficulty levels. Three-way mixed ANOVA [Factors: difficulty level (high, medium, low)* response side (left, right)*age (young, elderly)] revealed significant effect for difficulty level, $\mathrm{F}(2,70)=10.93, \mathrm{p}<0.01$, and an interaction between difficulty level, response side, and age, $F(2,82)=8.15, p<0.01$. Further analysis with pairwise comparisons using Fisher's LSD indicated that the largest number of errors occurred at the high $(\mathrm{M}=47)$ difficulty level and the smallest at the low $(\mathrm{M}=38)$ difficulty level [high-low $(\mathrm{p}<0.01)$; medium-low $(\mathrm{p}<0.01)$ ]. At the low difficulty level, the elderly responded more correctly $(\mathrm{M}=43)$ than the young $(\mathrm{M}=32)(\mathrm{p}=0.03)$. At the high difficulty level, the young responded more to the left $(\mathrm{M}=58)$ than to the right $(\mathrm{M}=34)$, paired sample $\mathrm{t}(24)=2.82, \mathrm{P}=0.01$, whereas elderly responded more to the right $(\mathrm{M}=59)$ than to the left $(\mathrm{M}=38)$, though not significantly, paired sample $\mathrm{t}(19)=-1.95, \mathrm{p}=0.06$. Independent $\mathrm{t}$-tests showed that the young and the elderly differed in their left responses $(\mathrm{M}=59, \mathrm{M}=38$, respectively), $\mathrm{t}(43)=-2.57, \mathrm{p}=0.01$, and also in their right side $\operatorname{responses}(\mathrm{M}=34, \mathrm{M}=58$, respectively), $\mathrm{t}(43)=3.63, \mathrm{p}<0.01$.

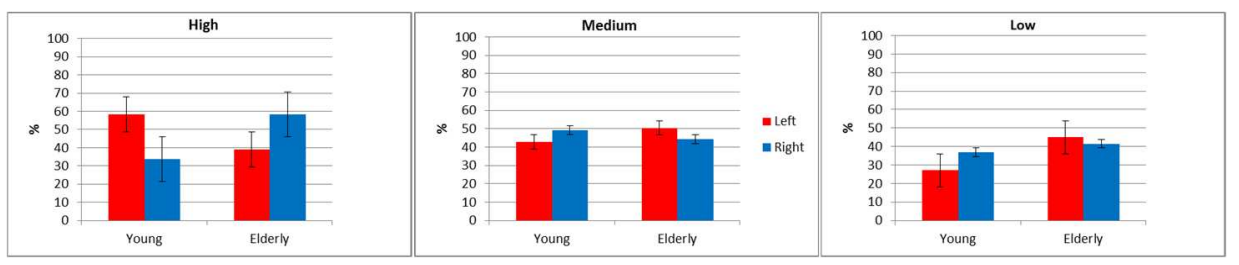

Figure 6. Erroneous unilateral responses to the bilateral auditory stimuli by the two age groups at the three difficulty levels, high(ISI=500-1000 ms), medium(ISI=700-1200 $m s)$, and low(ISI=1000-2000 ms).

\subsection{Visual stimuli}

\section{Correct responses}

Fig. 7 shows correct responses of the two age groups at the three difficulty levels in the three visual stimulus conditions. A three-way mixed ANOVA [Factors: stimulus condition(left, bilateral, right)*difficulty level(high, medium, low)*age(young, elderly)] revealed significant main effects for difficulty level, $\mathrm{F}(2,58)=92.45, \mathrm{p}<0.01$; stimulus 
condition, $\mathrm{F}(2,67)=7.37, \mathrm{p}<0.01 ;$ and age $\mathrm{F}(1,40)=21.09, \mathrm{p}<0.01$. The young outperformed the elderly. Pairwise comparisons of the difficulty levels using Fisher's LSD tests showed that the smallest number of correct responses were obtained at high $(\mathrm{M}=46)$ difficulty level, more at the medium $(\mathrm{M}=63)$ and the largest number at the low difficulty level $(\mathrm{M}=75)$ [high-medium $(\mathrm{p}<0.01)$, high-low $(\mathrm{p}<0.01)$, mediumlow $(\mathrm{P}<0.01)]$. Pairwise comparisons of the stimulus conditions showed that participants gave more correct responses to the right $(M=63)$ than to the left $(M=61)$ $(\mathrm{P}<0.01)$ or than to the bilateral stimuli $(\mathrm{M}=59)(\mathrm{p}<0.01)$.

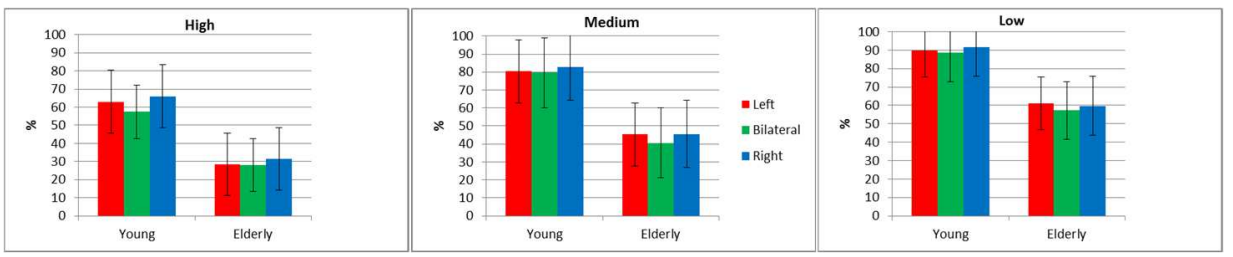

Figure 7. Correct responses to the three visual stimulus conditions, left, bilateral, and right, by the two age groups at the three difficulty levels, high(ISI=500-1000 ms), medium(ISI=700-1200 ms), and low(ISI=1000-2000 ms).

\section{$R T S$}

Fig. 8 shows the RTs at the three difficulty levels in the three stimulus conditions by the two age groups. Three-way mixed ANOVA [Factors: stimulus condition (left, bilateral, right)*difficulty level (high, medium, low)*age(young, elderly)] revealed significant main effects for difficulty level, $\mathrm{F}(2.72)=12.24, \mathrm{p}<0.01$, stimulus condition $\mathrm{F}(2,72)=11.39, \mathrm{p}<0.01$; and age, $\mathrm{F}(1,36)=16.24, \mathrm{p}<0.01$. The young reacted faster than the elderly.

Pairwise comparisons wish Fisher's LSD tests showed that the RTs were shortest at the high $(\mathrm{M}=511)$, longer at the medium $(\mathrm{M}=537)$ and longest at the low $(\mathrm{M}=546)$ difficulty levels [high-low $(\mathrm{p}<0.01)$, high-medium $(\mathrm{p}<0.01)$ ]. Pairwise comparisons of the stimulus conditions showed that participants reacted faster to the right $(\mathrm{M}=515)$ than to the left $(\mathrm{M}=537)(\mathrm{P}<0.01)$ or than to the bilateral stimuli $(\mathrm{M}=541)(\mathrm{p}<0.01)$.

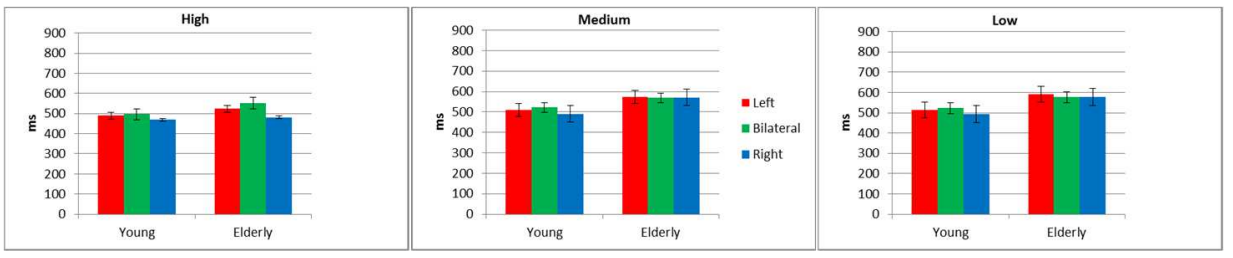

Figure 8. Reaction times to the three visual stimulus conditions, left, bilateral, and right, by the two age groups at the three difficulty levels, high(ISI=500-1000 ms), medium(ISI=700-1200 ms), and low(ISI=1000-2000 ms).

Erroneous unilateral responses to bilateral stimuli 
Fig. 9 shows erroneous unilateral responses of the age groups to three stimulus conditions at three difficulty levels. Three-way mixed ANOVA [Factors: difficulty level (high, medium, low)*response side (left, right)*age (young, elderly)] showed significant main effects for difficulty level, $\mathrm{F}(2,75)=40.35, \mathrm{p}<0.01$, response side, $\mathrm{F}(1,40)=$ $16.45, \mathrm{p}<0.01$, and age, $\mathrm{F}(1,40)=6.80, \mathrm{p}=01$. In general, more responses were made to the right than to the left. Elderly made more errors than young. Pairwise comparisons of the difficulty levels using Fisher's LSD showed that the largest number of erroneous responses were given at the high $(\mathrm{M}=49)$ difficulty level, a smaller number at the low $(\mathrm{M}=24)$ and the smallest at the medium $(\mathrm{M}=41)$ difficulty levels [high-medium $(\mathrm{P}<$ $0.1)$, high-slow $(\mathrm{P}<0.1)$, medium-slow $(\mathrm{P}<0.1)]$.

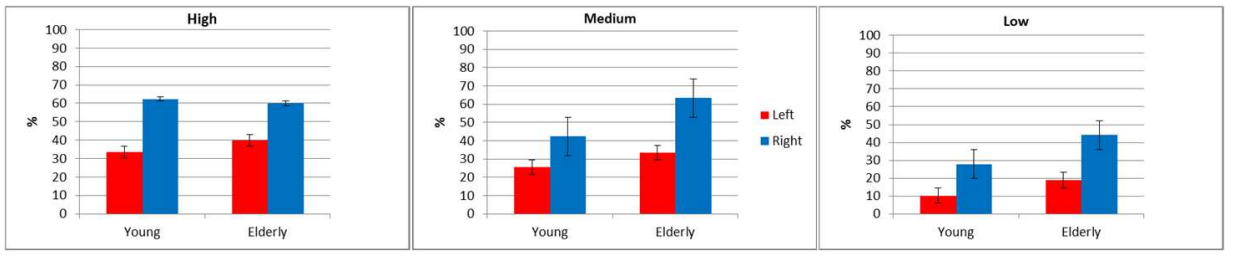

Figure 9. Erroneous unilateral responses to the bilateral stimuli by the two age groups at the three difficulty levels, high(ISI=500-1000 ms), medium(ISI=700-1200 ms), and low $(I S I=1000-2000 \mathrm{~ms})$.

\section{Discussion}

The results demonstrated the rather equal performance level of the two right-handed age groups in the virtual driving task, and the preference of the right side stimuli (significant for auditory) especially by the elderly participants. Also a slight superiority of the young over the elderly participants in their perceptual/attentional performance level while driving was obtained. The most unexpected finding was the simultaneous occurrence of rightward bias in spatial perception and attention and the leftward bias in driving errors. This was evident in both age groups but slightly more prominent in the elderly. The expected finding was the rightward bias in the perceptual and attentional capacity, which was most evident under larger cognitive load. Distinct rightward bias was now found for both the visual and the auditory spatial stimuli (cf. [2, 3] where the phenomenon was more distinct for auditory than visual stimuli).

A curious feature in driving was the bias in lane border crossings to the left. We have to focus on this phenomenon when more data is gathered. If it is true and more specifically, if it proves to be a stronger phenomenon among the older drivers, then the questions rises whether it has something to do with right-handedness and the spatial perceptual-attentional bias to the right by our present participants. We have originally suggested that the rightward spatial perceptual/attentional bias seen in our previous laboratory measurements $[2,3]$ is due to the functional lateralization of the normal and especially normally aging brain [4]. Furthermore, it has been shown previously that patients with hemispatial neglect, the pathological rightward bias of perception and attention due to injury of the right hemisphere attention mechanisms, have a bias to contralesional (left) collisions [6,7] when navigating a powered wheelchair. This is in contrast with the same patients in [7] study showing a rightward bias when walking, which reveals the task dependency of the spatial bias. Indeed, our preliminary findings 
indicate that there may be a connection between rightward bias in spatial perception and attention and the leftward bias in steering a vehicle in the normal and normally aging right-handed population. The positioning of the vehicle on the road (on the lane, between other vehicles) has been shown to be one of the key skills declining with aging $[8,9]$. These real-world phenomena can be reliably demonstrated even with a simple virtual system introduced in the present study.

The right-side bias found with the virtual system was expected since the phenomenon has been demonstrated in previous laboratory studies with both auditory [2] and visual [3] linguistic and nonlinguistic stimuli. In contrast to those previous studies, in the present results the bias in visual modality was an even more distinct that that in the auditory modality. This phenomenon needs further attention while more data, and possibly under driving tasks with different demands, is gathered. If it proves to be real it may indicate that the visuomotor driving task may induce changes in the processing of visual information, i.e. both tasks load the same visuo-spatial system, thus resulting in more bias in visual spatial perception.

The simultaneous driving/spatial perception/attention task works well. Not many (elderly) participants interrupted the task, and also errors were obtained in both age groups. As revealed by the performance of the young and elderly at lowest and highest difficulty levels, we feel that it is justified and necessary to have the different difficulty levels in the tasks. Because of the varying number of each stimulus modality and condition within the 2 min session, we already have modified the system into one, where the exact number of each stimulus condition is predetermined by the experimenter, and the session duration is at least $2 \mathrm{~min}$. As an alternative one could also think of a system which begins at low difficulty level, but then increases the difficulty level continuously until a clear reduction in performance level (e.g., 75\%) is reached.

The present low-cost system is designed for testing and evaluation purposes, but it can be used also as a template for training programs in the future. In the future versions also rear-view mirrors, pedals (plus gears), and turn-lights will be included in the system.

The relative amount of elderly population is growing at accelerating speed, and simultaneously an outstanding growth is seen in the traffic. This produces increasing demands to the driving skills and overall cognitive perceptual and attentional skills of the aging drivers, and thus means for extra training in safe environments must be developed. Recently very promising results have been reported related to the effects by training in the virtual environments on the working memory and executive functions (see e.g. $[10,11]$ which indeed are in focus in traffic behavior.

\section{Acknowledgement}

We would like to thank the funding agency, Finnish Funding Agency for Technology and Innovation (Tekes), The Traffic Safety Committee of Insurance Companies (VALT), Henry Ford Foundation, our academic and industrial partners, and the experts from the Turku Game Lab (TUAS) Finland. We also thank all the test subjects who took part in the experiments. 


\section{References}

[1] Takio F, Koivisto M, Jokiranta L, Rashid F, Kallio J, Tuominen T, Laukka S J, Hämäläinen $\mathrm{H}$ : The effect of age on attentional modulation in dichotic listening. Developmental Neuropsychology, Vol, 34, pp. 225-239, 2009 DOI:10.1080/87565640902805669

[2] Takio F, Koivisto M, Laukka S J., \& Hämäläinen H: Auditory rightward spatial bias varies as a function of age. Developmental Neuropsychology, Vol. 36, pp. 367- 387, 2011. DOI:10.1080/87565641.2010.549984

[3] Takio F, Koivisto M, Tuominen T, Laukka S J, Hämäläinen H: Visual rightward spatial bias varies as a function of age. Laterality: Asymmetries of Body, Brain and Cognition, e-pub, 1-24, 2012 DOI:10.1080/1357650X.2011.628675

[4] Takio F, Koivisto, M, \& Hämäläinen, H: The influence of executive functions on spatial biases varies during the lifespan. Developmental Cognitive Neuroscience, Vol. 10, 170-180, 2014. DOI:10.1016/j.den.2014.09.004

[5] Luimula M, Besz A, Pitkäkangas P, Suominen T, Smed J, Izullah, FR, and Hämäläinen H: Virtual Evaluation Tool in Driving Inspection and Training. in Proceedings of the 5th IEEE Conference on Cognitive Infocommunications, Gyor, Hungary, pp. 57-60, 2015 DOI: 10.1109/CogInfoCom.2015.7390564

[6] Punt TD, Kitadono K, Hulleman J, Humphreys, GW, Riddoch, MJ: From both sides now: crossover effects influence navigation in patients with unilateral neglect. Journal of Neurology, Neurosurgery, and Psychiatry, Vol. 79, pp. 464466, 2008 DOI:10.1136/jnnp.2007.129205

[7] Turton A J, Dewar SJ, Lievesley A, O'Leary K, Gabb J, \& Gilchrist, I D: Walking and wheelchair navigation in patients with left visual neglect. Neuropsychological Rehabilitation, Vol. 19, pp. 274-290, 2009

DOI: $0.1080 / 09602010802106478$

[8] Di Stefano M, MacDonald W: Assessment of older drivers: Relationships among on-road errors, medical conditions, and test outcomes. Journal of Safety Research, Vol. 34, pp. 415-429, 2003

DOI:10.1016/j.jsr.2003.09.010

[9] Baldock MRJ, Berndt A, Mathias, JL: The functional correlates of older drivers' on-road driving test errors. Topics in Geriatric Rehabilitation, Vol. 24, pp. 204223, 2008

DOI:10.1097/01.TGR.0000333754.90550.b5

[10] Anguera JA, Boccanfuso J, Rintoul JL, Al-Hashimi O, Faraji F, Janowich J, Gazzaley A: Video game training enhances cognitive control in older adults. Nature, 5 01, pp. 97-101, 2013

DOI: $10.1038 /$ nature12486

[11] Casutt G, Theill N, Martin M, Keller M, Jäncke L: The drive-wise project: driving simulator training increases real driving performance in healthy older drivers. Frontiers in Aging Neuroscience 6:85, 2014.

DOI:10.3389/fnagi.2014.00085 